\title{
Cultivated Limbal Stem Cell Transplantation-The Surgical Technique
}

\author{
Virender S Sangwan, MBBS, MS
}

Head of Cornea Service, and Associate Director, LV Prasad Eye Institute, Hyderabad

\begin{abstract}
Limbal stem cell deficiency (LSCD) is characterized by loss of limbal palisades of Vogt, ingrowth of conjunctiva on to the cornea, and recurrent or persistent epithelial defects of the cornea. This results in pain, watering, decrease in vision, and light sensitivity. LSCD is most commonly caused by ocular injury, immune-mediated ocular surface diseases, and aniridia. A variety of surgical techniques have been described to treat LSCD, but cultivated limbal stem cell transplantation seems the most promising. In this technique a small biopsy is harvested from healthy limbus, either of the same eye or the other eye. The limbal tissue is shredded into small pieces and seeded on to de-epithelialized human amniotic membrane. The human corneal epithelial medium is used to grow the cells. Twelve to 14 days of culture gives enough cells for clinical transplantation.
\end{abstract}

\section{Keywords}

Ocular surface, limbal stem cells, cultivated limbal stem cell transplantation, LSCD, surgical

Disclosure: The author has no conflicts of interest to declare.

Received: May 1, 2011 Accepted: January 4, 2012 Citation: US Ophthalmic Review, 2012;5(1)22-6 DOI: 10.17925/USOR.2012.05.01.22

Correspondence: Virender S Sangwan, MBBS, MS, Head of Cornea Service, and Associate Director, LV Prasad Eye Institute, Kallam Anji Reddy Campus, LV Prasad Marg, Banjara Hills, Hyderabad 500 034, India. E: vsangwan@Ivpei.org

The primary function of the ocular surface is to provide clear vision when the eye is open, as the anterior surface of the cornea contributes to more than two-thirds of the total refractive power of the eye. To achieve this, while maintaining comfort and preventing microbial invasion, the ocular surface must be covered by a stable tear film. The mechanism by which ocular surface health is insured is built into the intimate relationship between ocular surface epithelium and the preocular tear film. There is a constant turnover of ocular surface epithelium as it is replenished by adult stem cells found in conjunctiva and at the limbus. Damage to the ocular surface caused by chemical, thermal, or mechanical injuries, or by immune-mediated diseases, results in limbal stem cell deficiency (LSCD). This is manifested as vascularization and chronic inflammation of the cornea, ingrowth of conjunctiva on to the cornea (conjunctivalization), corneal opacification, and persistent or recurrent corneal epithelial defects. This leads to decrease or loss of vision in the affected eye with pain, redness, watering, and light sensitivity.

There has been considerable progress in the recent past in understanding the physiology and the molecular and cellular biology of the ocular surface. This has also opened a new chapter in regenerative medicine with the use of tissue engineering. ${ }^{1-3}$ Various scientific discoveries in the past two decades have led to the identification of the limbal location of corneal epithelial stem cells and the role they play in regenerating the corneal epithelium. ${ }^{1,4-7}$ Simultaneously, various surgical techniques of limbal transplantation using cadaveric ${ }^{2}$ or live related-donor tissues have evolved for the treatment of bilateral LSCD., 9 However, these methods have met with limited success and allografts require immunosuppression for an indefinite period to avoid rejection..$^{10,11}$ However, long-term systemic immunosuppression involves the risk of serious eye and systemic complications, apart from being a significant economic burden. For unilateral total or partial LSCD direct or cultivated autologous limbal transplantation works very well. With direct limbal stem cell transplantation there is a need to transplant the 3-6 o'clock healthy limbus from the contralateral eye. With this technique there is the potential for donor site LSCD.

Novel techniques of ex vivo cell culture have allowed us to treat LSCD with a better surgical approach that requires very little donor tissue, thus minimizing the risk to the donor site and increasing the possibility of obtaining autologous donor tissue from a small uninvolved area of the limbus. ${ }^{12-14}$ This method is called autologous cultivated limbal epithelial transplantation (CLET). The idea came from the successful culture of human keratinocytes (epidermal cells from skin) onto a feeder layer of non-proliferative 3T3 fibroblasts. The cells may secrete some growth factors that are conducive to growth of the keratinocytes. This idea was incorporated in the culture of corneal epithelial cells, and the first report of this method was by Pellegrini et al. ${ }^{12}$ in 1997 where two patients with alkali burn were successfully transplanted with ЗТ3 fibroblast feeder-cultured autologous corneal epithelium, derived from mechanically and enzymatically disintegrated $2 \times 2 \mathrm{~mm}$ limbal biopsy taken from the other healthy eye. Since then, there have been many modifications of this original technique. A few such methods using different culture techniques and carriers have been reported in the literature..$^{12,13,15-17}$ 
Figure 1: Schematic Representation of an Autologous Cultivated Limbal Stem Cell Transplantation

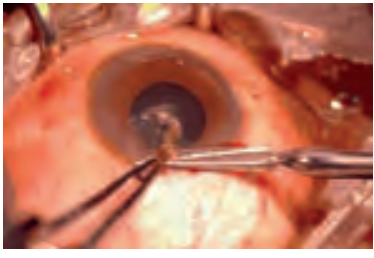

Biopsy being taken from contralateral healthy limbus

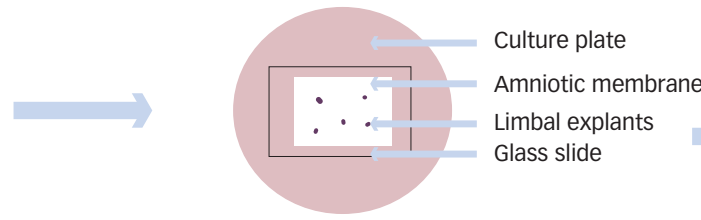

Limbal explants shredded into small pieces and implanted on de-epithelialized amniotic membrane
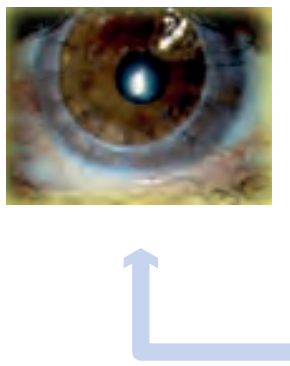

Slit lamp photograph of the same patient six months after penetrating keratoplasty (10 months post-cultured limbal epithelial transplantation)

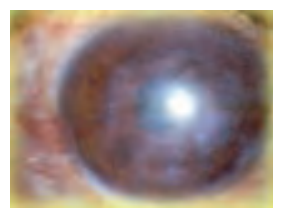

Same eye, post-cultured limbal epithelial transplantation, showing stable corneal surface with improved clarity

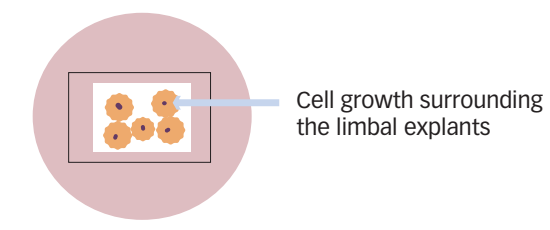

Confluent growth obtained surrounding the explants

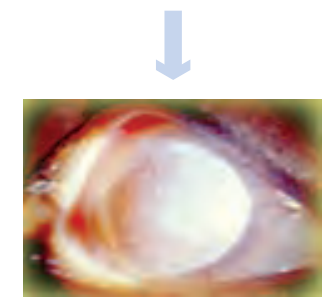

Slit lamp photograph of an eye with total limbal stem cell deficiency

\section{The Methodology of Limbal Biopsy and Culture of Explanted Tissue}

We have earlier reported our surgical techniques for CLET. ${ }^{18}$ After obtaining informed consent from the patients or guardians, limbal biopsy is performed on the healthy contralateral eye or a healthy area of the same eye. The procedure involves careful dissection of a $2 \times 2 \mathrm{~mm}$ piece of limbal epithelium with $0.5 \mathrm{~mm}$ into clear corneal stromal tissue at the limbus under strict aseptic conditions under local or general anesthesia, depending on the patient's preference and age. The limbal tissue that contains limbal epithelial cells at the pigmented line (palisades of Vogt) and a part of the corneal stroma are excised. If LSCD is bilateral, then tissue can be taken from live related-donor or cadaveric sources. A study in our laboratory ${ }^{19}$ has shown that fresh limbal tissue with $100 \%$ viability is preferable to preserved cadaveric limbal tissue with $51.6 \%$ viability for culture. The tissue is transported in human corneal epithelium medium to the tissue culture laboratory where, under strict aseptic conditions, the donor limbal tissue is shredded into small pieces (see Figure 1). A similar parallel culture is prepared as a back-up. Human amniotic membrane (HAM), prepared and preserved by our eye bank, is used as a carrier. HAM measuring $2.5 \times 5 \mathrm{~cm}$ is de-epithelialized using $0.25 \%$ trypsin and ethylenediamine tetra-acetic acid (EDTA) solution for 30 minutes. Ours is a submerged explant culture system without the use of any feeder cell layer. ${ }^{20}$

Another culture system is that of cell suspension, where epithelial cells are isolated by enzymatic digestion of limbal tissue and expanded using the 3T3 fibroblast cells as the feeder cell layer. The air-lift technique used by other groups involves the culturing of cells for a period of three weeks and then lowering the amount of culture medium so as to provide an air-water interface for another week. This promotes stratification of the epithelial cell cultures and also improves intercellular junctions. Though superior in terms of stem cell content, ${ }^{16,21}$ however, this method is more expensive and technology-intensive. Our system is simple and cost-effective, and only a monolayer is required for transplantation. We have shown that stratification happens in vivo after transplantation and have provided clinical in vivo confocal microscopic and histological evidence of stratification of the transplanted monolayer of cultivated epithelium. ${ }^{22}$

Earlier, we had used human corneal epithelium medium with $10 \%$ fetal bovine serum to nurture the culture. However, for the past seven years we have been using autologous serum instead of fetal bovine serum. The human corneal epithelium medium is prepared using $9.7 \mathrm{~g} / \mathrm{l}$ modified Eagle medium with the addition of $16.2 \mathrm{~g} / \mathrm{l} \mathrm{Ham}$ 's F12 serum, $0.01 \mathrm{mg} / \mathrm{l}$ epidermal growth factor, $0.25 \mathrm{mg} / \mathrm{l}$ insulin, $0.1 \mathrm{mg} / \mathrm{l}$ cholera toxin, and hydrocortisone. The medium is filtered using a $0.22 \mathrm{~mm}$ membrane filter and a vacuum pump. The sterile medium is supplemented with $10 \%$ autologous serum at the time of use. The culture is incubated at $37{ }^{\circ} \mathrm{C}$ with $5 \%$ carbon dioxide and $95 \%$ air. The growth is monitored daily under phase contrast microscopy and the medium changed every alternate day. The culture is terminated when the monolayer of cells growing from the explants becomes confluent, usually in 10-14 days. Cultures covering more than a $50 \%$ area of $2.5 \times 5 \mathrm{~cm}$ HAM are considered adequate for clinical use.

\section{Transplantation of Cultivated Limbal Epithelial Cells on the Amniotic Membrane Pre-operative Management}

A careful clinical examination is required for all potential CLET patients. The ocular adnexa should be examined for any defects; if they are found to have lid notch or defects, these should be repaired prior to CLET. The patients should not have surface anesthesia as is found in patients 
with herpes zoster ophthalmicus or seventh nerve paralysis. If there is underlying immune-mediated inflammation, as seen in ocular cicatricial pemphigoid (OCP) or Stevens-Johnson syndrome (SJS), then it should be treated with appropriate therapy before performing CLET. If the LSCD is due to chemical injury, enough time should be allowed before attempting ocular surface reconstruction so that inflammation subsides and the chances of survival of transplanted epithelial cells improve. The visual potential of the eye should be carefully assessed before carrying out the surgery, especially in children where there is the possibility of amblyopia.

\section{Surgical Technique}

The surgery is performed under local or general anesthesia, based on the patient's choice as well as the patient's age. In younger children general anesthesia is preferred. A drop of epinephrine $(1: 1,000)$ is instilled in the conjunctival cul-de-sac prior to pannus excision to decrease bleeding during the procedure. Dissection of ocular surface pannus is started 2-3 $\mathrm{mm}$ behind the limbus if this landmark is still visible. Blunt-tip spring scissors or conjunctival scissors are used to initiate the dissection by lifting the pannus and dissection is continued all around the limbus with a \#15 blade on a Bard Parker ${ }^{\circledR}$ handle. A combination of sharp and blunt dissection from the periphery toward the central area is carried out to clean the cornea. It is important to pay attention to the thickness of the corneal tissue, which may be variable due to the effects of chemicals or disease processes, and it is vital not to perforate the cornea. Any accidental perforation, which can be identified by softening of the globe, should be repaired either by suturing or by application of fibrin tissue sealant. If the perforation is too large to be repaired, lamellar or penetrating keratoplasty (PKP) must be performed along with cultivated limbal stem cell transplantation. Perforation is uncommon if the surgeon respects the tissue planes. The ocular surface is thus cleared of the fibrovascular pannus and hemostasis is achieved by judicious use of bipolar cautery. Adhesions between the eyeball and lids (symblephara) are released, taking due care not to injure the extra-ocular muscles and other tissues. The fornix is reconstructed using amniotic membrane, and a fornix-forming suture, if indicated. At this stage the corneal thickness is assessed clinically and by pachymetry. If the recipient corneal thickness is less than $300 \mu \mathrm{m}$, a lamellar corneal graft is carried out to restore the thickness of the cornea; this will help support the epithelialization of the ocular surface following cultivated epithelial transplantation. At this stage, the cultivated limbal epithelium on HAM is brought onto the ocular surface, cell side up, aseptically, and the membrane containing the cultured cells is gently spread over the cornea, as well as the limbus, without damaging or dislodging the cells. The area of maximum number of visible explants with surrounding cells is kept at the center of the cornea and the peripheral part of the membrane is trimmed using blunt-tip scissors. There are two ways of anchoring the composite of membrane and cells onto the ocular surface. If sutures are used, 10-0 monofilament nylon material is preferred. The tissue should be anchored using circumferential interrupted sutures at the limbus. The peripheral edge of the membrane is sutured to the conjunctiva with 8-0 polyglactin (VicrylTM) sutures. Usually six to eight interrupted sutures are enough to secure the membrane and care should be taken to avoid any folds or wrinkles of the membrane. If they are present, the tissue should be stretched and unfolded, holding the peripheral part of the membrane. The central part of the membrane, which has maximum cellular growth, is never touched because it is critical for re-epithelialization. If there is inadvertent needle perforation, recognized by softening of the eye, then the suture should be taken at another location. Needle perforations do not usually require repair. If sutureless surgery is planned (see Figure 2), the fibrin tissue sealant (Tisseel ${ }^{\mathrm{TM}}$ Kit; Baxter AG, Vienna, Austria) is applied to the ocular surface using a tuberculin syringe with 27-gauge needle. Both components are applied, one after the other, and the membrane with grown cells is brought onto the ocular surface and spread as explained above. Excess membrane is trimmed and the peripheral part of the membrane is ironed out to avoid wrinkles or folds of the membrane. The membrane is touched with a surgical sponge to test if the adhesion is sufficient to hold the tissue in place. Sometimes there may be fluid accumulation under the membrane during the post-operative period. To avoid this complication, two small holes are made in the membrane at the limbus five and seven o'clock positions so the fluid is drained out.

At the conclusion of the surgery, one drop of $2.5 \%$ povidone-iodine is instilled; use of a bandage contact lens is optional. Some surgeons recommend using a bandage contact lens for the first few weeks, to avoid loss of cells due to blinking. For the same reason, some surgeons prefer to induce ptosis by injecting botulinum toxin into the orbicularis oculi. We do not use any of these techniques because we believe that there are firm adhesions between the membrane and the growing cells. The technique described above is for total LSCD where fibrovascular pannus covers the entire ocular surface. If there is focal or sectoral deficiency, the surgical technique is tailored to cover the defect created by removal of abnormal tissue. Either sutures or fibrin glue can be used to anchor the membrane with cultivated cells to the ocular surface.

\section{Post-operative Follow-up and Treatment}

Following CLET, all patients are treated with prednisolone acetate $1 \%$ eye drops, eight times a day, tapered to once a day in 5-6 weeks, and ciprofloxacin hydrochloride $0.3 \%$ eye drops, four times a day, for one week. The ciprofloxacin eye drops are continued if an epithelial defect is present or as long as the bandage contact lens is used. If a limbal allograft is performed, the patient should be given systemic immunosuppression, as has been reported earlier. ${ }^{17}$ Patients are examined on post-operative day one, at one week, two weeks, five weeks and monthly thereafter for six months. Thereafter, follow-up is tailored to the success or failure of the procedure. If this is successful and does not require further interventions, follow-up is every six months for three years and annually thereafter. For failed surgery, the process could be repeated or appropriate alternative therapy offered. Each examination includes a complete history, noting of new ocular or systemic symptoms, and complete evaluation of the recipient and donor sites, as well as any signs of neovascularization or surface instability. Sutures are removed when indicated (loose or vascularized). Epithelialized sutures are left indefinitely. Some CLET patients may require PKP or deep anterior lamellar keratoplasty if vision does not improve with CLET alone.

\section{Outcome of Cultivated Limbal Epithelium Transplantation}

Clinical outcome is determined by noting the improvement in ocular surface stability, as judged by non-recurrence of conjunctivalization and the absence of epithelial defects. There are several case reports and small case series reported in the literature using this technique. One earlier case report by Nakamura et al. ${ }^{23}$ in 2004 described the success in 


\section{Figure 2: Pannus Excision and Cultivated Epithelium-Amniotic Membrane Composite Grafting}
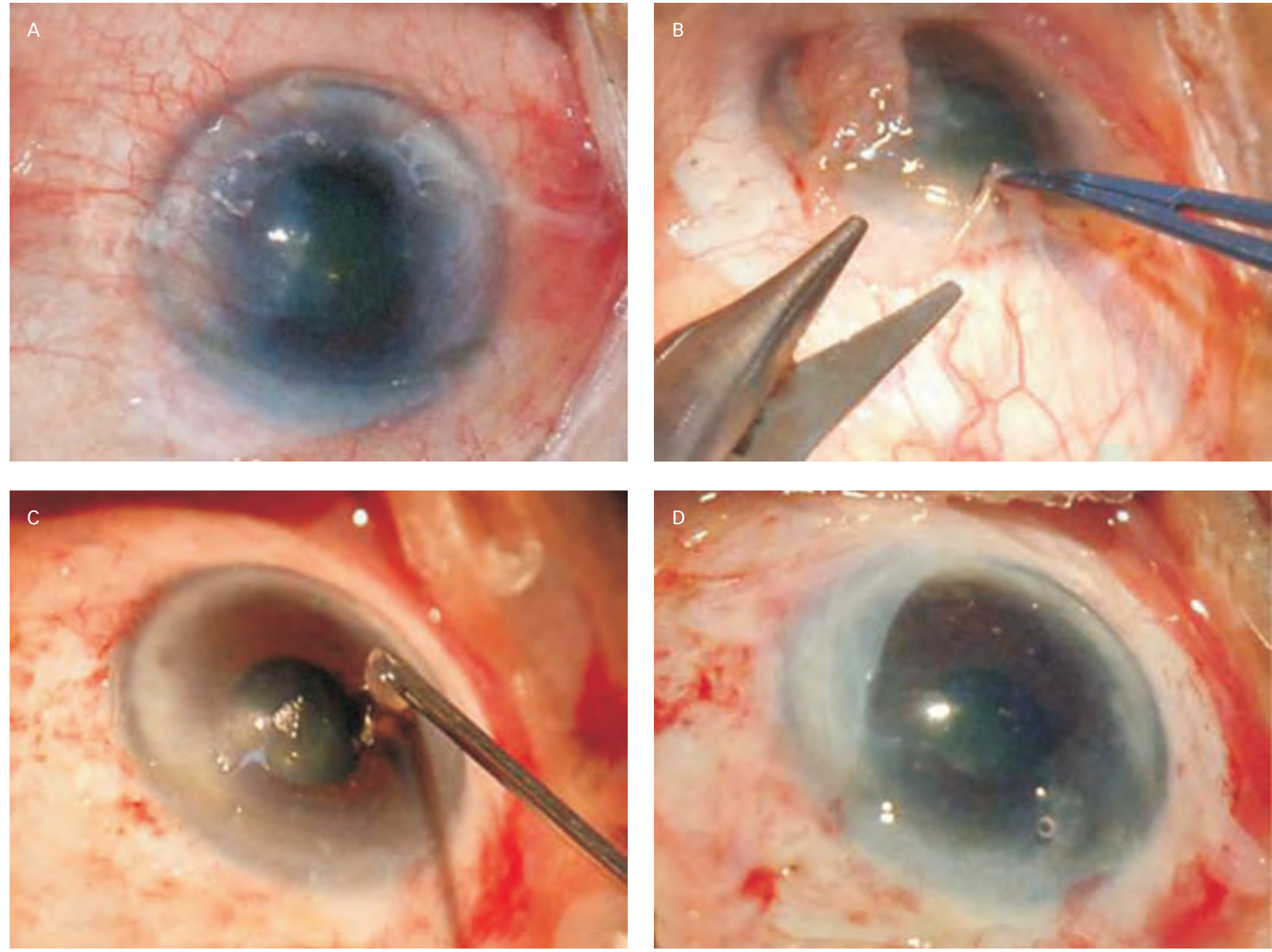

A: Pre-operative photo showing total limbal stem cell deficiency (LSCD); B: Photo showing beginning of excision of fibrovascular pannus; C: Application of fibrin glue on the corneal surface after complete removal of pannus; D: Completed surgery for amniotic membrane with cultivated stem cells on it. Monolayer of cultivated epithelium cannot be seen in this photo; it can only be seen with a phase contrast microscope.

culturing autologous limbal epithelial cells from a minimal limbal biopsy from the uninjured eye and subsequent transplantation to a patient with LSCD due to chemical injury. Thereafter, we reported the largest series of autologous CLET in 2006. ${ }^{24}$ Eighty-eight eyes of 86 patients underwent autologous CLET between 2001 and 2003; alkali burn was the most common cause of LSCD ( $n=56)$, and 61 of these 88 eyes had total LSCD. Success was achieved in $73.1 \%$ of cases (63.3-82.9 $95 \%$ confidence interval) and best corrected visual acuity (BCVA) on the snellen chart improved from $17 / 88$ (21.8 \%) to $41 / 88$ (52.6\%) of eyes having functional ambulatory vision (>20/200) post-operatively. We have reported early results of PKP following CLET. Fifteen of 125 patients underwent PKP following CLET, at a mean interval of seven months. Fourteen of the 15 eyes had a successful corneal graft with stable corneal epithelium.

Koizumi et al. reported transplantation of allogenic cultivated corneal limbal epithelium from donor corneas. ${ }^{25}$ The cells were cultivated on denuded amniotic membrane with a 3T3 fibroblast feeder cell layer. The air-lift technique was used to achieve stratification of the epithelium. Thirteen eyes with total LSCD from 11 patients were selected for the procedure. The cultivated epithelium was transplanted to the diseased eye. Simultaneous lamellar keratoplasty was performed in five patients with associated stromal scarring. All the recipients were started on an immunosuppression regime. After 48 hours, all of the 13 eyes showed regained epithelial integrity. On follow-up for six months, 10 of the 13 eyes (77 \%) showed improved BCVA, a gain of at least two lines in the Snellen chart. However, three eyes experienced epithelial rejection. This finding underscores the importance of proper immunosuppression for the allograft technique.

In conclusion, LSCD can be effectively treated by autologous or allogeneic cultivated limbal epithelial transplantation. The major advantage of this technique is that it is very safe for the donor eyes and this has made it increasingly popular for the treatment of unilateral LCSD. Additionally, in patients with bilateral but asymmetrical involvement, a small area of healthy limbus in either eye is sufficient to generate transplantable sheets 


\section{Surgery}

of cultivated limbal epithelium which can then be used to treat both eyes. Not only is surgical technique of both limbal biopsy and transplantation simple and sutureless, our technique of limbal cultivation is also rapid and completely avoids the use of animal derived products in cell culture. Over the last decade, significant advances in the reliability of cultivation techniques and reporting of the excellent long-term outcomes of clinical transplantation have established CLET as the preferred surgical technique for the treatment of LSCD, wherever appropriate laboratory facilities exist. Further refinement and popularization of this technique may eventually lead to elimination of needless corneal blindness due to LSCD.
1. Sangwan VS, Tseng SCG, New perspectives in ocular surface disorders: An integrated approach for diagnosis and management Indian J Ophthalmol, 2001:49(3):153-68.

2. Tsubota K, Satake $Y$, Kaido M, et al., Treatment of severe ocular-surface disorders with corneal epithelial stem-ce transplantation, N Engl J Med, 1999;340(22):1697-1703.

3. Sangwan VS, Vemuganti GK, Singh S, Balasubramanian D, Successful reconstruction of damaged ocular outer surface in humans using limbal and conjunctival stem cell culture methods, Biosci Rep, 2003;23(4):169-74.

4. Zieske JD, Bukusoglu G, Yankauckas MA, Characterization of potential marker of corneal epithelial stem cells, Invest Ophthalmol Vis Sci, 1992;33(1):143-52.

5. Cotsarelis G, Cheng SZ, Dong G, et al., Existence of slow-cycling limbal epithelial basal cells that can be preferentially stimulated to proliferate: implications on epithelial stem cells, Cell, 1989:57(2):201-9.

6. Schermer A, Galvin S, Sun TT, Differentiation-related expression of a major $64 \mathrm{~K}$ corneal keratin in vivo and in culture suggests limbal location of corneal epithelial stem cells, I Cell Biol, 1986;103(1):49-62

7. Kruse FE, Stem cells and corneal epithelial regeneration, Eye (Lond), 1994;8(2):170-83

8. Rao SK, Rajagopal R, Sitalakshmi G, Padmanabhan P, Limbal allografting from related live donors for corneal surface reconstruction, Ophthalmology, 1999;106(4):822-8.

9. Daya SM, Ilari FA, Living related conjunctival limbal allograft for the treatment of stem cell deficiency, Ophthalmology, 2001:108(1):126-33.

10. Solomon A, Ellies P, Anderson DF, et al., Long-term outcome of keratolimbal allograft with or without penetrating keratoplasty for total limbal stem cell deficiency, Ophthalmology, 2002;109(6):1159-66.

11. Ilari L, Daya SM, Long-term outcomes of keratolimbal allograft for the treatment of severe ocular surface disorders, Ophthalmology, 2002:109(7):1278-84.

12. Pellegrini G, Traverso CE, Franzi AT, et al., Long-term restoration of damaged corneal surfaces with autologous cultivated corneal epithelium, Lancet, 1997;349(9057):990-3.

13. Tsai RJ, Li LM, Chen JK, Reconstruction of damaged corneas by transplantation of autologous limbal epithelial cells, N Engl J Med, 2000;343(2):86-93.

14. Schwab IR, Reyes M, Isseroff RR, Successful transplantation of bioengineered tissue replacements in patients with ocular surface disease, Cornea, 2000;19(4):421-6.

15. Meller D, Pires RT, Tseng SC, Ex vivo preservation and expansion of human limbal epithelial stem cells on amniotic membrane cultures, Br J Ophthalmol, 2002;86(4):463-71.

16. Koizumi N, Cooper LJ, Fullwood NJ, et al., An evaluation of cultivated corneal limbal epithelial cells, using cell-suspension culture, Invest Ophthalmol Vis Sci, 2002;43(7):2114-21.

17. Sangwan VS, Vemuganti GK, Iftekhar G, et al., Use of autologous cultured limbal and conjunctival epithelium in a patient with severe bilateral ocular surface disease induced by acid injury: a case report of unique application, cornea, 2003;22(5):478-81.

18. Sangwan VS, Matalia HP, Vemuganti GK, et al., Early results of penetrating keratoplasty after cultivated limbal epithelium transplantation, Arch Ophthalmol, 2005;123(3):334-40.

19. Vemuganti GK, Kashyap S, Sangwan VS, Singh S, Ex-vivo potential of cadaveric and fresh limbal tissues to regenerate cultured epithelium, Indian J Ophthalmol, 2004;52(2):113-20.

20. Fatima A, Sangwan VS, Iftekhar G, et al., Technique of cultivating limbal derived corneal epithelium on human amniotic membrane for clinical transplantation, I Postgrad Med, 2006;52(4):257-61.

21. Zhang $X$, Sun $H$, Tang $X$, et al., Comparison of cell-suspension and explant culture of rabbit limbal epithelial cells, Exp Eye Res, 2005;80(2):227-33.

22. Fatima A, vemuganti GK, Iftekhar $\mathrm{G}$, et al., In vivo survival and stratification of cultured limbal epithelium, Clin Experiment Ophthalmol, 2007;35(1):96-8.

23. Nakamura T, Inatomi T, Sotozono C, et al., Successful primary culture and autologous transplantation of corneal limbal epithelial cells from minimal biopsy for unilateral severe ocular surface disease, Acta Ophthalmol Scand, 2004;82(4):468-71.

24. Sangwan VS, Matalia HP, Vemuganti GK, et al., Clinical outcome of autologous cultivated limbal epithelium transplantation, Indian J Ophthalmol, 2006;54:29-34.

25. Koizumi N, Inatomi T, Suzuki T, et al., Cultivated corneal epithelial stem cell transplantation in ocular surface disorders, ophthalmology, 2001;108(9):1569-74. 